\title{
HIGH-ORDER ENTROPY-BASED CLOSURES FOR LINEAR TRANSPORT IN SLAB GEOMETRY*
}

\author{
CORY D. HAUCK ${ }^{\dagger}$
}

\begin{abstract}
We compute high-order entropy-based $\left(M_{N}\right)$ models for a linear transport equation on a one-dimensional slab geometry. We simulate two test problems from the literature: the twobeam instability and the plane-source problem. In the former case, we compute solutions for systems up to order $N=6$; in the latter, up to $N=15$. The most notable outcome of these results is the existence of shocks in the steady-state profiles of the two-beam instability for all odd values of $N$.
\end{abstract}

Key words. Particle transport, maximum entropy, moment closures.

AMS subject classifications. 82C70, 82D75, 94A17.

\section{Introduction}

In transport and kinetic theory, moment models are used to reduce the size of the state space required for a kinetic description while still maintaining basic features of a kinetic model. They do so by replacing the velocity component of phase space by a finite number of velocity moments. Moment models are commonly derived using an approximate reconstruction of the kinetic description from these moments. The reconstruction prescribes a closure, i.e. a recipe for expressing the moment model as a closed system of the retained moments. Entropy-based methods specify this reconstruction as the solution to a constrained, convex optimization problem. In many situations the cost functional for the optimization problem is directly related to the kinetic entropy of the system. In other cases it simply enforces physically relevant features. The benefit of the entropy approach is a reduced model which retains fundamental properties from the kinetic formalism not found in traditional moment models - properties such as hyperbolicity, entropy dissipation, and positivity. The main disadvantage of the entropy approach is that, unlike traditional moment models, the entropy-based kinetic reconstruction can rarely be expressed as an analytic function of the given moments. Thus the optimization problem must be solved numerically via the associated dual problem. This can increase computational costs significantly.

Recent advances in both analysis and implementation of entropy-based methods have been made in several application areas. For gas dynamics, the formal properties of entropy-based models were elucidated in [26]. However, it is also known $[17,20,21,38]$ that the defining optimization problem in this case is ill-posed. As a result, alternative approaches are currently being pursued which regularize the problem in some suitable fashion; see [15] and references therein. For charge transport in semiconductors, the issue of ill-posedness also exists for the so-called parabolic band approximation [29, p.69]. However, for experimental dispersion relations or for more realistic approximations, like the Kane dispersion relation [3, p.3], the optimization

${ }^{*}$ Received: February 1, 2010; accepted (in revised version): May 28, 2010. Communicated by Pierre Degond.

${ }^{\dagger}$ Computational Mathematics Group, Computer Science and Mathematics Division, Oak Ridge National Laboratory, Oak Ridge, TN 37831 USA (hauckc@ornl.gov).

This research was sponsored by the Office of Advanced Scientific Computing Research; U.S. Department of Energy. This work began at Los Alamos National Laboratory, which is managed by Los Alamos National Security, LLC under Contract No. DE-AC52-06NA25396, and was completed at the Oak Ridge National Laboratory, which is managed by UT-Battelle, LLC under Contract No. De-AC05-00OR22725. 
problem is well-posed [22]. Indeed recent simulations [37] have shown reasonable agreement with Monte-Carlo results.

In the field of radiative transport, moments are typically taken with respect to functions of the angular component of the microscopic particle velocity only. A general theoretic framework was laid out in [10], but most computational results have been limited to the so-called Minerbo, or $M_{1}$, model or variations thereof $[5,11,12,31]$, which track moments of the kinetic distribution with respect to polynomials up to degree one. The reason for this limitation is that, beyond the $M_{1}$ model, the defining optimization problem is especially hard to solve for highly anisotropic distributions that commonly occur in transport applications. A recent and notable advance in this direction is the recent work in $[32,41]$, where the authors simulate the $M_{2}$ model (the next model in the hierarchy, which tracks moment with respect to polynomials up to degree two) for one-dimensional slab geometries. This simulation is done using a clever procedure to bypass the optimization problem completely. The most powerful aspect of this approach is the ability to handle beam-like distributions with relative ease. The procedure is based on a look-up table and hence becomes less practical when more moments are added (either by increasing the polynomial degree further or by adding more dimensions). Interpolation can also be used to reduce the number of entries in the look-up table, but exactly how such an interpolation effects hyperbolicity of the moment system is unclear. Thus, one must consider attacking the optimization problem head-on. In the future, it is likely that a combination of the two methods will be most effective.

In spite of the heavy computational overhead of solving difficult optimization problems, the entropy-based approach still has practical merit for large-scale, massively parallel computations that one might see in a complex multi-physics application. This is due to the emerging paradigm in parallel computing in which data transfernot floating point operations - is the bottleneck to efficient computation [1,30,42]. In particular, though the optimization requires many function evaluations, the solver for updating the moment equations uses the same amount of data transfer between computational cells as it would for a conventional, algebraic closure.

The purpose of this paper is not to develop an efficient optimization algorithm, but rather to gain some insight about what can be gained by going to higher-order closures. We consider entropy-based models, including and beyond $M_{1}$ and $M_{2}$, for the simple case of linear transport in slab geometries, utilizing the Maxwell-Boltzmann entropy as the cost functional in our optimization problem. The moment equations in this setting are formulated in Section 2. In Section 3, we present the kinetic scheme used to implement them. In Section 4, we present numerical results for two test cases found elsewhere in the literature. Section 5 is devoted to conclusions and discussion.

\section{Entropy-based moment equations}

We assume a distribution of particles of a single unit speed which are absorbed by or isotropically scattered off of a background material medium with slab geometry. We let $x \in\left(x_{1}, x_{2}\right)$ be the scalar coordinate along the axis perpendicular to the slab; $\mu \in[-1,1]$ is the cosine of the angle between the $x$-axis and the direction of particle travel; $t \geq 0$ is time; and for any integrable function $g=g(\mu)$,

$$
\langle g\rangle \equiv \int_{-1}^{1} g(\mu) d \mu .
$$

The state of the particles is characterized by a non-negative function $F(x, \mu, t)$. 
The material is characterized by non-negative variables $\sigma_{\mathrm{s}}(x), \sigma_{\mathrm{a}}(x)$, and $\sigma_{\mathrm{t}}(x)=$ $\sigma_{\mathrm{s}}(x)+\sigma_{\mathrm{a}}(x)$ which are the scattering, absorption, and total cross-sections, respectively, of the material. Each cross-section is the reciprocal of the mean-free-path between particle interactions of a given type. The function $S$ is an external source, which in general may depend on properties of the material. However, for the test cases in Section 4, we will take $S=0$.

In this setting the relevant transport equation for $F$ takes the form $[6,27,36]$

$$
\partial_{t} F+\mu \partial_{x} F+\sigma_{\mathrm{t}} F=\frac{\sigma_{\mathrm{s}}}{2} \rho+S,
$$

where $\rho:=\langle F\rangle$ is the particle concentration. The transport equation is supplemented by boundary and initial conditions

$$
\begin{aligned}
F\left(x_{1}, \mu, t\right) & =F_{1}(\mu, t), \quad \mu>0, \\
F\left(x_{2}, \mu, t\right) & =F_{2}(\mu, t), \quad \mu<0, \\
F(x, \mu, 0) & =F_{0}(x, \mu) .
\end{aligned}
$$

Here $F_{0}, F_{1}$, and $F_{2}$ are given.

Moment equations for (2.2) are typically based on angular averages with respect to Legendre polynomials. To derive an order $N$ system, we collect the first $N+1$ Legendre polynomials into a vector-valued function $\mathbf{m}:[-1,1] \rightarrow \mathbb{R}^{N+1}$. Exact equations for the moments $\mathbf{u}(x, t)=\left[u_{0}, \ldots, u_{N}\right]^{T}:=\langle\mathbf{m} F(x, \cdot, t)\rangle$ are found by multiplying the transport Equation (2.2) by $\mathbf{m}$ and integrating over all angles. This gives the system

$$
\partial_{t} \mathbf{u}+\partial_{x}\langle\mu \mathbf{m} F\rangle+\sigma_{\mathrm{t}} \mathbf{u}=\frac{\sigma_{\mathrm{s}}}{2} Q \mathbf{u}+\mathbf{s},
$$

where

$$
Q_{l k}:=\delta_{l k} \delta_{l, 0} \quad \text { and } \quad \mathbf{s}:=\langle\mathbf{m} S\rangle
$$

Boundary conditions for the moment system are not so easily expressed since kinetic data is only given for values of $\mu$ which correspond to incoming data. Indeed, the issue of proper boundary conditions remains an open question, although some progress has been made for linear systems $[23-25,35] .{ }^{1}$

The moment system (2.4) is closed by substituting the approximation

$$
F(x, \mu, t) \simeq \mathcal{F}(\mathbf{u}(x, t), \mathbf{m}(\mu))
$$

into (2.4), where the reconstruction $\mathcal{F}$ satisfies the consistency relation

$$
\langle\mathbf{m} \mathcal{F}(\mathbf{u}, \mathbf{m})\rangle=\mathbf{u} .
$$

The resulting system takes the form

$$
\partial_{t} \mathbf{u}+\partial_{x}\langle\mu \mathbf{m} \mathcal{F}(\mathbf{u}, \mathbf{m})\rangle+\sigma_{\mathrm{t}} \mathbf{u}=\frac{\sigma}{2} Q \mathbf{u}+\mathbf{s}
$$

The question of whether (2.8) can accurately capture the dynamics of the original transport equation depends heavily on the details of $\mathcal{F}$.

\footnotetext{
${ }^{1}$ For computations presented later in the paper, we prescribe boundary conditions that are reasonable, but not entirely consistent with the kinetic boundary conditions.
} 
Entropy-based methods generate a closure by prescribing $\mathcal{F}$ as the solution of a constrained, convex optimization problem. To be more specific, given a strictly convex mapping $\eta: \mathbb{R} \ni z \mapsto \eta(z) \in \mathbb{R}, \mathcal{F}$ solves

$$
\begin{aligned}
\operatorname{minimize} & \langle\eta(f)\rangle \\
\text { subject to } & \langle\mathbf{m} f\rangle=\mathbf{u} .
\end{aligned}
$$

If a solution exists, it takes the form [26]

$$
\mathcal{F}(\mathbf{u}, \mathbf{m})=G_{\hat{\boldsymbol{\alpha}}(\mathbf{u})}:=\eta_{y}^{*}\left(\hat{\boldsymbol{\alpha}}(\mathbf{u})^{T} \mathbf{m}\right)
$$

where $\eta^{*}: \mathbb{R} \ni y \mapsto \eta^{*}(y) \in \mathbb{R}$ is the Legendre dual of $\eta, \eta_{y}^{*}$ is its derivative, and the vector $\hat{\boldsymbol{\alpha}}: \mathbb{R}^{N+1} \ni \mathbf{u} \mapsto \hat{\boldsymbol{\alpha}}(\mathbf{u}) \in \mathbb{R}^{N+1}$ solves the dual problem

$$
\operatorname{minimize}_{\boldsymbol{\alpha} \in \mathbb{R}^{N+1}}\left\{\left\langle\eta^{*}\left(\boldsymbol{\alpha}^{T} \mathbf{m}\right)\right\rangle-\boldsymbol{\alpha}^{T} \mathbf{u}\right\} .
$$

In particular, first-order optimality conditions for (2.11) recover the consistency relation (2.7):

$$
\left\langle\mathbf{m} \eta_{y}^{*}\left(\hat{\boldsymbol{\alpha}}^{T} \mathbf{m}\right)\right\rangle=\mathbf{u}
$$

Furthermore, it is straightforward to show (following arguments in $[10,26]$, for example) that the moment system dissipates the strictly convex entropy $h(\mathbf{u}):=\left\langle\eta\left(G_{\hat{\boldsymbol{\alpha}}(\mathbf{u})}\right)\right\rangle$ and that, when written in terms of $\hat{\boldsymbol{\alpha}}$, the entropy-based moment system is symmetric hyperbolic.

Different choices of $\eta$ lead to different closures. In gas dynamics applications, one typically uses the Maxwell-Boltzmann entropy; for fermions, the Fermi-Dirac entropy; for bosons (as in photon transport), the Bose-Einstein entropy. In some cases, the choice of entropy is not directly related to the physics of the problem. For example, the choice $\eta(z)=z^{2} / 2$ leads to the well-known $P_{N}$ equations $[7,27,36]$. For the purposes of this paper, we assume classical particles with a slight variation of the Maxwell-Boltzmann entropy

$$
\eta(z)=z \log (z)-z
$$

and Legendre dual $\eta^{*}(y)=e^{y}$. In this case, the exponential form ensures that the reconstruction is always a positive function.

\section{Implementation with a kinetic scheme}

We implement the moment equations from the previous section using a kinetic scheme $[8,9,16,33,34]$. In a conventional approach, one first takes moments of the kinetic equations and then finds a valid spatial discretization of the resulting moment system. With a kinetic scheme, these steps are reversed: one first determines a valid discretization of the kinetic equation and then takes moments of this discretization. The benefit of this approach is that it avoids the direct computation of eigenvalues and (approximate) Riemann solvers for the entropy-based moment systems, which generally cannot by computed by hand.

3.1. Description of the scheme. Let $\left\{x_{j}\right\}_{j=1}^{J}$ be an evenly spaced set of mesh points which serve as the centers of cells $I_{j}=\left(x_{j-1 / 2}, x_{j+1 / 2}\right)$ of width $\Delta x$. For simplicity of notation, we assume here that all material cross-sections are constant. ${ }^{2}$

\footnotetext{
${ }^{2}$ Our test problems in the next section will all satisfy this assumption, but there is certainly no limitation that requires it.
} 
A semi-discrete, finite-volume formulation of the transport equation is derived by integration in $x$ over each $I_{j}$, giving

$$
\partial_{t} F_{j}+\mu \frac{F_{j+1 / 2}-F_{j-1 / 2}}{\Delta x}+\sigma_{\mathrm{t}} F_{j}=\frac{1}{2} \sigma_{\mathrm{s}} \rho_{j}+S_{j} .
$$

Here $j$ subscripts adorn cell averages (in space) and $j \pm 1 / 2$ subscripts denote pointwise values at cell edges, which are approximated by upwinding: for $\mu>0$, information comes from the left; for $\mu<0$, it comes from the right. When applied to (3.1), upwinding gives

$$
\begin{aligned}
\partial_{t} F_{j} & +\max (\mu, 0) \frac{F_{j+1 / 2, \ell}-F_{j-1 / 2, \ell}}{\Delta x} \\
& +\min (\mu, 0) \frac{F_{j+1 / 2, r}-F_{j-1 / 2, r}}{\Delta x}+\sigma_{\mathrm{t}} F_{j}=\frac{1}{2} \sigma_{\mathrm{s}} \rho_{j}+S_{j},
\end{aligned}
$$

where $F_{j+1 / 2, \ell}$ and $F_{j+1 / 2, r}$ are values on the right and left sides of the cell edge at $x_{j+1 / 2}$. A linear approximation of these values is

$$
F_{j+1 / 2, \ell}=F_{j}+\frac{\Delta x}{2} F_{j}^{\prime} \quad \text { and } \quad F_{j+1 / 2, r}=F_{j+1}-\frac{\Delta x}{2} F_{j+1}^{\prime},
$$

where $F_{j}^{\prime}$ is an approximation of the spatial gradient in cell $j$. We use the well-known minmod limiter function:

$$
F_{j}^{\prime}=\operatorname{minmod}\left\{\frac{F_{j}-F_{j-1}}{\Delta x}, \frac{F_{j+1}-F_{j-1}}{2 \Delta x}, \frac{F_{j+1}-F_{j}}{\Delta x}\right\} .
$$

The idea of the limiter is to suppress spurious oscillations in the solution and, in the context of the entropy closure, to ensure that the edge values for $F$ are non-negative.

To obtain an algorithm for $\mathbf{u}_{j}:=\left\langle\mathbf{m} F_{j}\right\rangle$ with the entropy-based closure, we simply integrate the discretization (3.1) of the kinetic equation against the vector $\mathbf{m}$ and replace $F_{j}$ by $G_{j}:=G_{\hat{\alpha}\left(\mathbf{u}_{j}\right)}$. This gives

$$
\begin{aligned}
\partial_{t} \mathbf{u}_{j} & +\frac{\left\langle\mu \mathbf{m}\left[G_{j+1 / 2, \ell}-G_{j-1 / 2, \ell}\right]\right\rangle_{+}}{\Delta x} \\
& +\frac{\left\langle\mu \mathbf{m}\left[G_{j+1 / 2, r}-G_{j-1 / 2, r}\right]\right\rangle_{-}}{\Delta x}+\sigma_{\mathrm{t}} \mathbf{u}_{j}=\frac{\sigma_{\mathrm{s}}}{2} Q \mathbf{u}_{j}+\mathbf{s}_{j},
\end{aligned}
$$

where plus/minus subscripts denote integration over positive/negative ranges of $\mu$ (so-called half fluxes), $Q$ is given in (2.5), and $\mathbf{s}_{j}=\left\langle\mathbf{m} S_{j}\right\rangle$.

Time integration of (3.5) depends on the type of problem. For marching to steady state, first-order explicit Euler is satisfactory. However, for transient solutions, we use the second-order, strong stability preserving, Runge-Kutta (SSP-RK2) method $[14,39]$. As a reminder to the reader, if we write the semi-discrete system $(3.5)$ in the abstract form

$$
\partial_{t} \overrightarrow{\mathbf{u}}=L(\overrightarrow{\mathbf{u}}),
$$

then the SSP-RK2 method takes the form

$$
\begin{aligned}
\overrightarrow{\mathbf{u}}^{(1)} & =\overrightarrow{\mathbf{u}}^{n}+\Delta t L\left(\overrightarrow{\mathbf{u}}^{n}\right), \\
\overrightarrow{\mathbf{u}}^{n+1} & =\frac{1}{2} \overrightarrow{\mathbf{u}}^{n}+\frac{1}{2} \overrightarrow{\mathbf{u}}^{(1)}+\frac{1}{2} \Delta t L\left(\overrightarrow{\mathbf{u}}^{(1)}\right) .
\end{aligned}
$$




\begin{tabular}{|l|l|l|}
\hline Parameter & Meaning & Value \\
\hline \hline MaxFunEvals & $\begin{array}{l}\text { Maximum number of function } \\
\text { evaluations allowed }\end{array}$ & 10000 \\
\hline MaxIter & Maximum number of iterations allowed & 10000 \\
\hline LargeScale & Use large-scale algorithm if possible & off \\
\hline Display & Level of display & notify \\
\hline TolFun & $\begin{array}{l}\text { Termination tolerance on the objective } \\
\text { function and gradient }\end{array}$ & $\epsilon \simeq 2.22 \times 10^{-16}$ \\
\hline TolX & Termination tolerance on $\mathrm{x}$ & $\epsilon \simeq 2.22 \times 10^{-16}$ \\
\hline GradObj & $\begin{array}{l}\text { Gradient for the objective function } \\
\text { defined by the user }\end{array}$ & on \\
\hline Hessian & $\begin{array}{l}\text { Hessian for the objective function } \\
\text { defined by the user }\end{array}$ & off \\
\hline
\end{tabular}

TABLE 3.1. Optimization parameters for fminunc. Parameters that are not given are set to default values.

3.2. Details and subtleties. In this subsection, we briefly discuss important details of the algorithm and some subtle difficulties. Most of these difficulties arise when solving the dual problem (2.11).

1. Numerical integration. The algorithm requires a quadrature rule to evaluate the half fluxes. We use a Gauss-Legendre quadrature with 20 quadrature points. Efficiency and accuracy can be increased by using an adaptive quadrature. However, any adaptive rule must still guarantee convergence and be integrated into the kinetic scheme. Work in this direction in on-going.

2. Optimization parameters. The entire algorithm is implemented in MATLAB. The bulk of the computation lies in the repeated solution of the dual problem (2.11), which we find using the built-in function fminunc. Parameters of interest for fminunc are set with the command optimset and are given in Table 3.1. All other parameters are set to default values. With the Hessian parameter set to off, fminunc approximates Hessians using finite differences of the gradient.

3. Difficulties with the optimization. The built-in function fminunc breaks down in regimes near the boundary of so-called realizability. A vector $\mathbf{u}$ is said to be realizable whenever it is the moment of a non-negative function of $\mu$. The set of realizable vectors forms an open set [21] and, roughly speaking, functions of $\mu$ which generate vectors near the boundary of this set are highly anisotropic and beam-like. For example, one can show that certain vectors on the boundary can only be generated by delta functions [32]. When the reconstruction $G_{\hat{\boldsymbol{\alpha}}}$ is highly anisotropic, solving the dual problem for $\hat{\boldsymbol{\alpha}}$ becomes quite difficult because the Hessian becomes singular. Such cases often occur around discontinuities, singularities, and voids in space. Thus, increasing the spatial resolution of the computation typically makes the optimization more difficult. In an effort to avoid the boundary of realizability, the test problems in the following sections are slight modifications of problems found in the literature. In particular, voids are replaced by an isotropic distribution $F_{\text {floor }}$, which is set to a small value. Even with this modification, fminunc often terminates prematurely - not because some tolerance criteria is satis- 
fied, but because the approximate Hessian no longer predicts a decrease in the function value. Although the error in approximating the nearly-singular Hessian can be fixed by supplying an exact Hessian, fminunc will often still terminate because the ceiling set by MaxFunEvals and MaxIter is broken. In order to be sure of reasonable accuracy in the solution of the dual problem, we compute the difference between the value $\mathbf{u}_{j}$ and the moment of the reconstruction $\left\langle\mathbf{m} G_{j}\right\rangle$. For all cases, we have found the maximum difference over the entirety of the calculation to be $O\left(10^{-8}\right){ }^{3}$ In the meantime, devising a more flexible optimization tool is the focus of ongoing research.

4. Behavior in diffusive regimes. The kinetic scheme is very inefficient in diffusive regimes, where $\sigma_{\mathrm{t}}$ is large. Indeed, the formal accuracy of the scheme in space is $O\left(\sigma_{\mathrm{t}} \Delta x^{3}\right)$ (see, for example, the second-order upwind analysis in $[19,28])$. Furthermore, because the scheme is explicit, $O\left(\Delta x / \sigma_{\mathrm{t}}, 1 / \sigma_{\mathrm{t}}\right)$ time steps are required for stability. Thus, in diffusive regimes, accuracy requirements dictate that the spatial and temporal mesh be small even if the solution profile varies on an $O(1)$ scale. Fortunately, the test cases which we will consider in the next section are not in the diffusive regime; instead $\sigma_{\mathrm{t}}$ is an $O(1)$ quantity. Even so, practical applications will eventually require that more robust algorithms be developed for diffusive or multi-scale problems. Such algorithms are the focus of future efforts.

5. Implementation of boundary conditions. As mentioned in Section 2, proper boundary conditions for moment equations are not known. For the kinetic scheme, we set (refer to (2.3))

$$
G_{1 / 2, \ell}\left(\mu_{k}, t\right)=F_{1}\left(\mu_{k}, t\right) \text { and } G_{J+1 / 2, r}\left(\mu_{k}, t\right)=F_{2}\left(\mu_{k}, t^{n}\right)
$$

for any $\mu_{k}$ in the quadrature set used to evaluate the integrals in (3.5). While reasonable, this is clearly not the only option. Moreover, in the context of gas dynamics, it has been shown [40] that this condition gives inaccurate results unless the mesh resolves the mean free path. Fortunately, this condition holds for the test problems we consider in the next section. One can also argue that, to be consistent with the moment approach, the kinetic data at the boundaries should be replaced by a reconstruction of the form $G_{\hat{\alpha}}$ - that is,

$$
G_{1 / 2, \ell}\left(\mu_{k}, t\right)=G_{\boldsymbol{\alpha}_{1 / 2, \ell}} \text { and } G_{J+1 / 2, r}\left(\mu_{k}, t\right)=G_{\boldsymbol{\alpha}_{J+1 / 2, r}},
$$

where the coefficients $\boldsymbol{\alpha}_{1 / 2, \ell}$ and $\boldsymbol{\alpha}_{J+1 / 2, r}$ are determined by $N+1$ moment constraints of the form

$$
\left\langle\phi_{l} G_{\boldsymbol{\alpha}_{J+1 / 2, r}}\right\rangle=\left\langle\phi_{l} G_{J+1 / 2, \ell}\right\rangle_{+}+\left\langle\phi_{l} F_{2}\right\rangle_{-}, \quad \phi_{l}=\phi_{l}(\mu), \quad l=0, \ldots, N
$$

on the right boundary, with similar conditions on the left. One could let $\phi_{l}=m_{l}$, as is done in [40], but this is not the only possibility.

\section{Numerical results}

In this section, we present results for two types of problems found in the literature: the two-beam instability and the plane source. For the former we are interested

\footnotetext{
${ }^{3}$ Although we have not done so, one could presumably increase the tolerance parameter TolFun to a value of this size to ensure proper termination of fminunc.
} 
primarily in steady-state solutions for a purely absorbing material, while for the latter we focus on transient solutions for a purely scattering material. All results are given in terms of the particle concentration $\rho \equiv u_{0} \equiv\langle F\rangle$.

4.1. Two-beam instability. The two-beam instability is a test of a closure's ability to handle multi-modal distributions. In this one-dimensional setting, the material slab is initially in an (almost) vacuum state. Particles enter the domain from the right and left boundary and a steady-state is reached. Under some conditions, the $M_{1}$ closure is unable to handle particles moving in opposing directions and, as a result, produces a shock in the steady-state profile $[5,11]$. It has been shown recently $[32,41]$ that, in some cases, the $M_{2}$ model and also versions of the $M_{1}$ model that use partial moments [12] can produce reasonably accurate results without this unphysical shock.

We implement the two-beam instability on a one-dimensional slab covering the interval $[-1,1]$ in space. The parameter values are

$$
\sigma_{\mathrm{t}}=\sigma_{\mathrm{a}}=2.0, \quad S=0 .
$$

Thus there is no scattering $\left(\sigma_{\mathrm{s}}=0\right)$ and no external source. The initial condition for the kinetic equations is (refer to (2.3))

$$
F_{0}(x, \mu)=F_{\text {floor }}=0.5 \times 10^{-4},
$$

and the boundary conditions are isotropic:

$$
F_{1}(\mu, t)=F_{2}(\mu, t)=0.5
$$

Boundary and initial conditions for the kinetic scheme are derived from these. The small, non-zero value for the initial condition is needed to maintain stability with respect to the dual problem (2.11), as discussed in the previous section. We march to steady-state using the kinetic scheme with forward Euler time discretization and time step $\Delta t=0.3 \Delta x$.

Results for the two-beam instability are given in Figure 4.1-4.6. For comparison, we include the exact steady-state concentration, $\rho_{\mathrm{ss}} \equiv\left\langle F_{\mathrm{ss}}\right\rangle$, where

$$
F_{\mathrm{SS}}(x, \mu)= \begin{cases}0.5 e^{\frac{-\sigma_{\mathrm{a}}(x+1)}{\mu}}, & \mu>0, \\ 0.5 e^{\frac{-\sigma_{\mathrm{a}}(x-1)}{\mu}}, & \mu<0,\end{cases}
$$

and the angular integral is computed with a 20-point Gauss-Legendre quadrature.

In Figure 4.1, we show solutions at time $t=5.0$, when the solution is essentially at steady-state. Roughly speaking, the solutions improve as $N$ increases, but the even and odd profiles are qualitatively different. The even solutions appear to converge from above near the boundary and from below near the center. They also appear to show a dip in the very center. The odd solutions, on the other hand, appear to converge from below near the boundary and from above near the center. They also show a noticeable shock, in which the concentration jumps up when moving from the boundary toward the interior: at $x= \pm 0.28$ for $M_{1}, x= \pm 0.32$ for $M_{3}$ and $x= \pm 0.26$ for $M_{5}$. The size of this shock decreases as $N$ increases. Previously it has been speculated that the reason for the $M_{1}$ shock was that the entropy reconstruction did not possess enough degrees of freedom to reproduce two opposing beams. However, these results point to something deeper, since the $M_{3}$ and $M_{5}$ solutions also display shocks. 


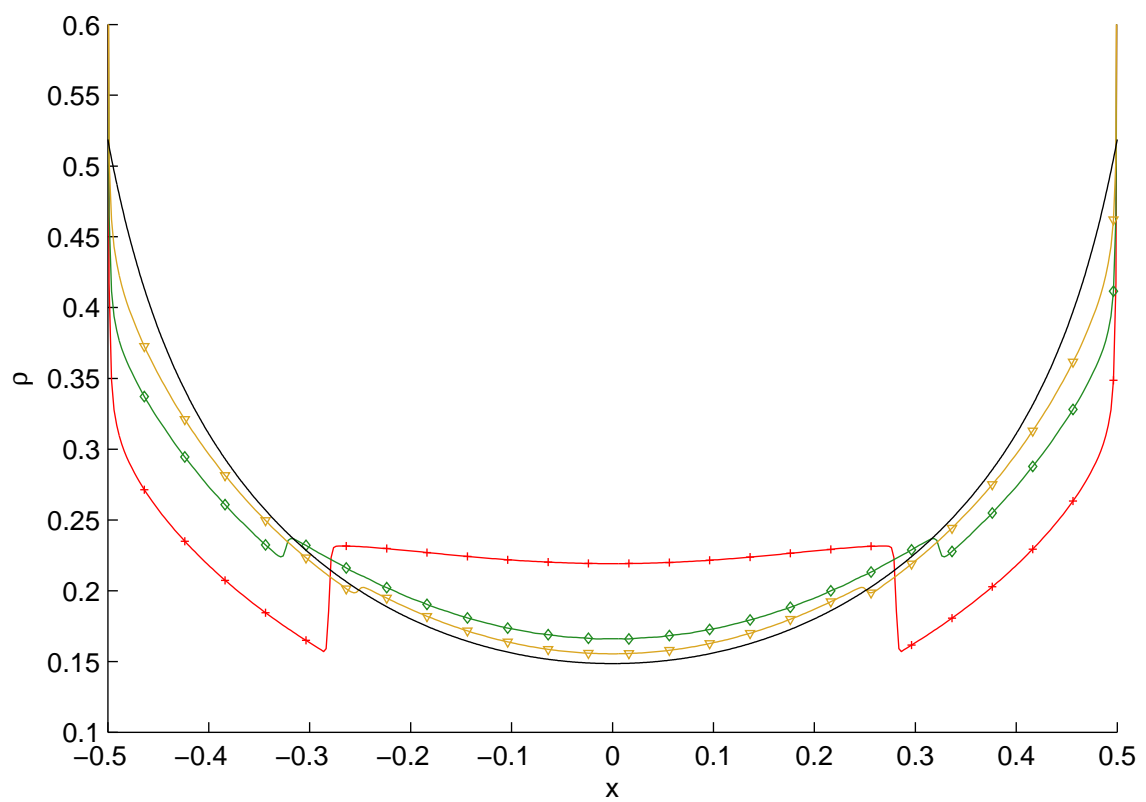

(a) Odd $N\left(M_{1}, M_{3}, M_{5}\right)$

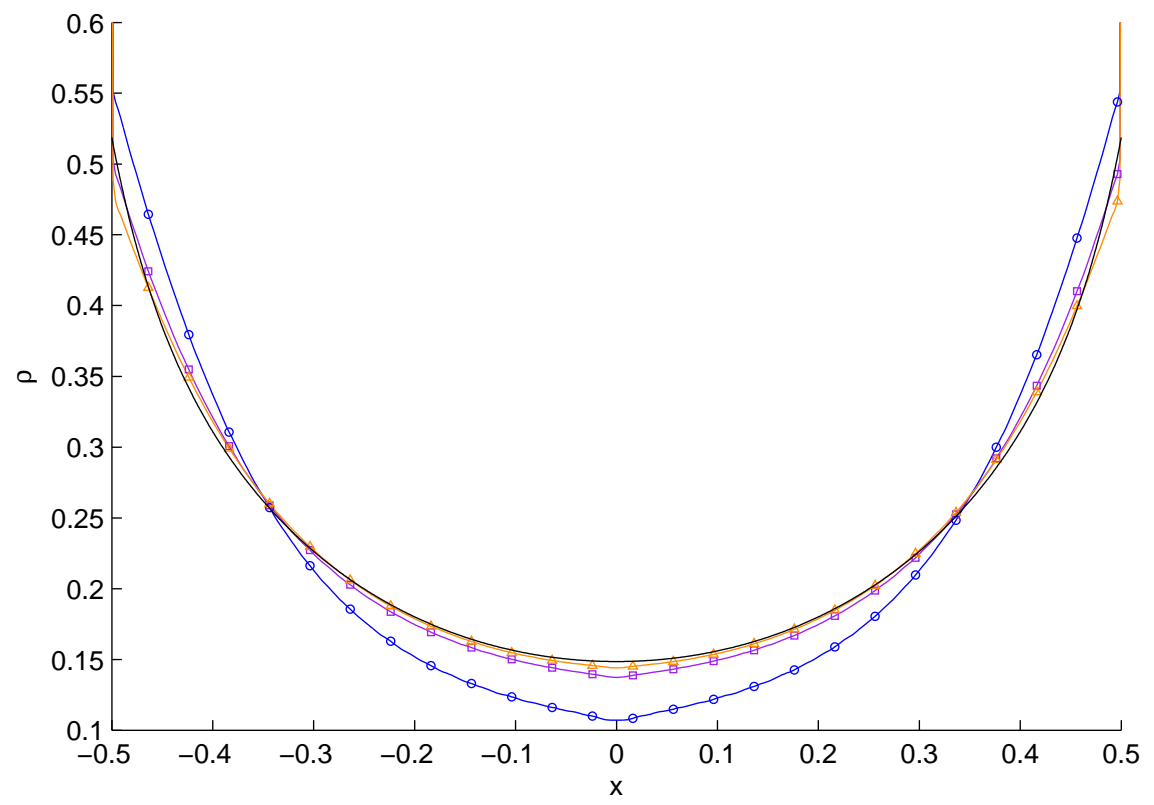

(b) Even $N\left(M_{2}, M_{4}, M_{6}\right)$

FIG. 4.1. Steady-state solutions for the two-beam instability. Red pluses are $M_{1} ;$ blue circles are $M_{2}$; green diamonds are $M_{3}$; purple squares are $M_{4} ;$ gold triangles are $M_{5}$; orange triangles are $M_{6}$. Solid, unadorned black line is the exact steady-state solution. The reader is referred to the online version for color interpretation. 


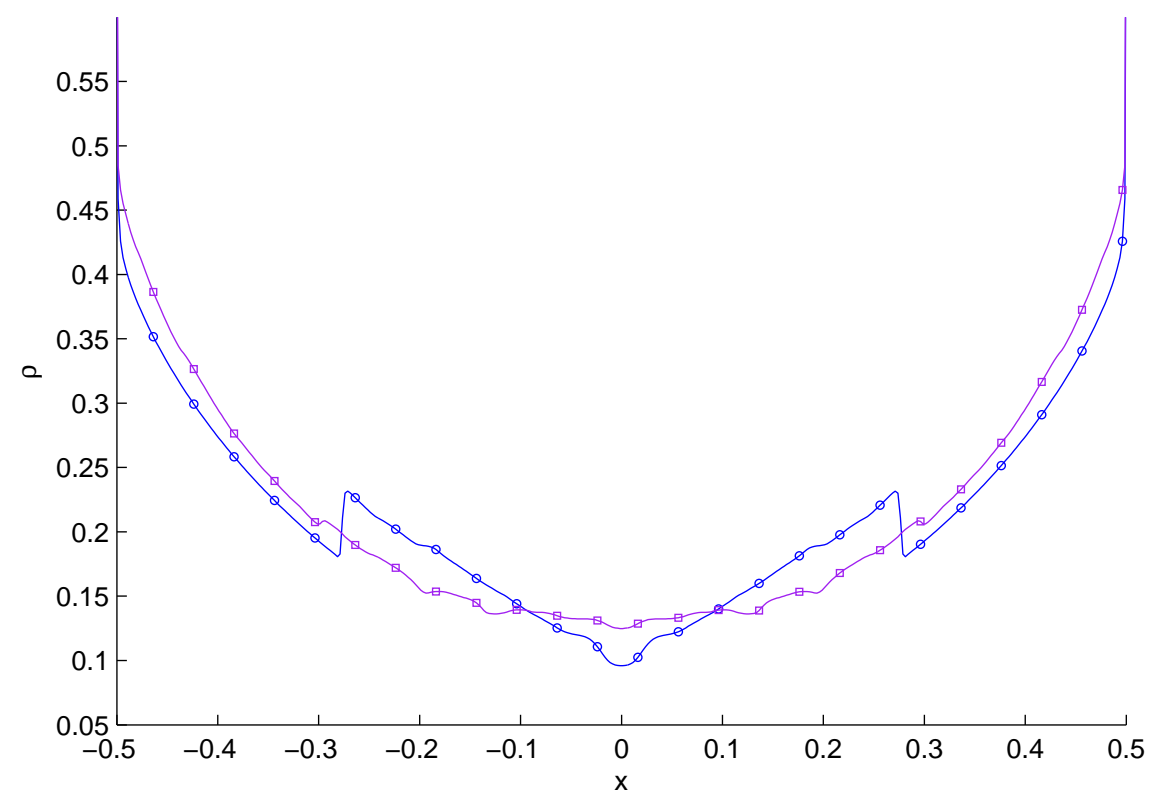

FIG. 4.2. Transient solutions at $t=1.0$. Blue circles are $M_{2}$; purple squares are $M_{4}$. The reader is referred to the online version for color interpretation.

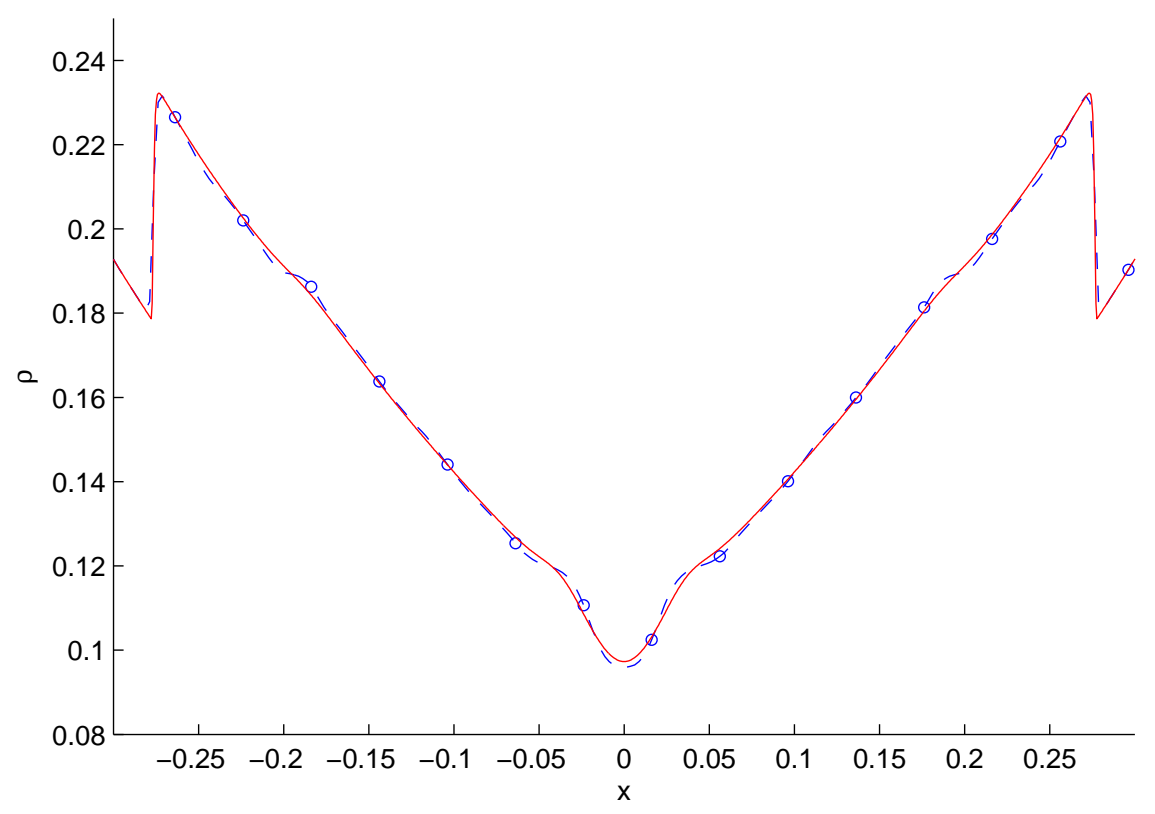

FIG. 4.3. $M_{2}$ solution for the two-beam instability at $t=1$. Dotted line with blue circles is the standard resolution (400 spatial cells and 20 quadrature points). The solid red line is high resolution (1600 spatial cells and 80 quadrature points). The reader is referred to the online version for color interpretation. 


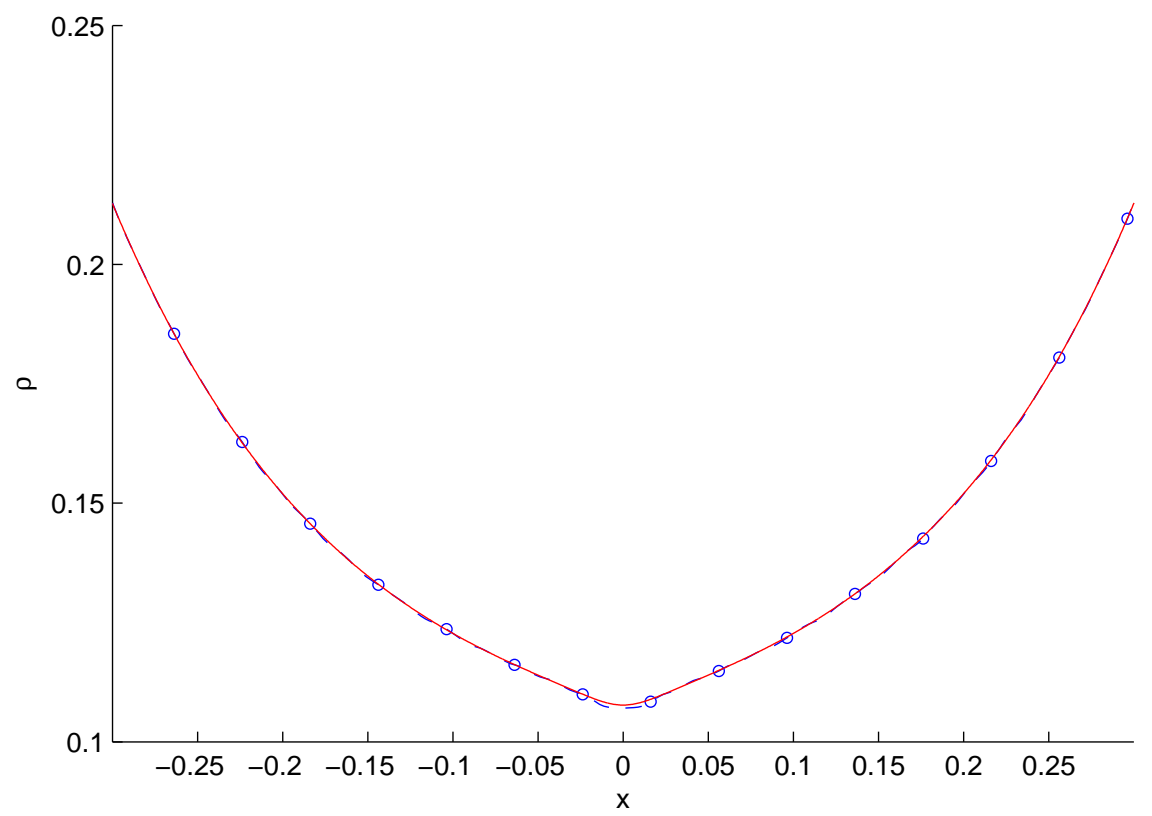

FIG. 4.4. Steady-state $M_{2}$ solution for the two-beam instability. Dotted line with blue circles is the standard resolution (400 spatial cells and 20 quadrature points). The solid red line is high resolution (1600 spatial cells and 80 quadrature points). The reader is referred to the online version for color interpretation.

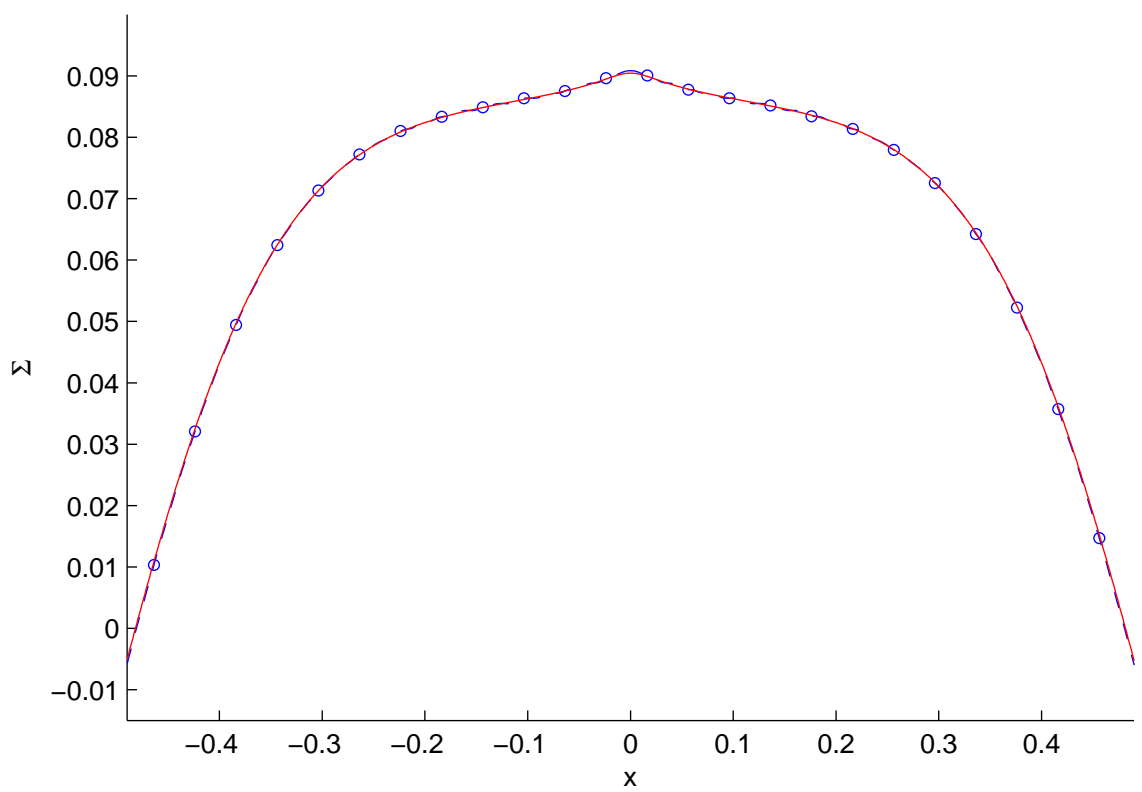

FIG. 4.5. Steady-state $M_{2}$ solution for the two-beam instability. Dotted line with blue circles is the standard resolution (400 spatial cells and 20 quadrature points). The solid red line is high resolution (1600 spatial cells and 80 quadrature points). The reader is referred to the online version for color interpretation. 


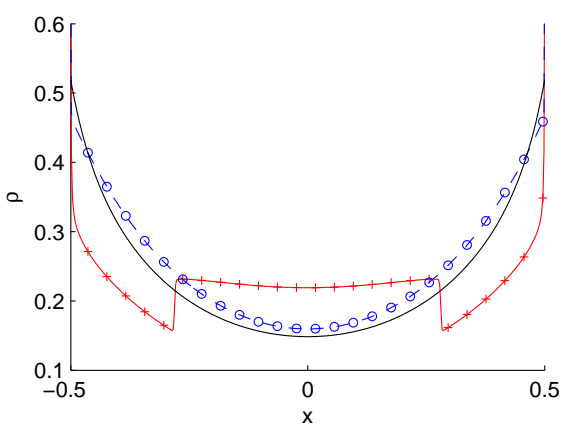

(a) $N=1$

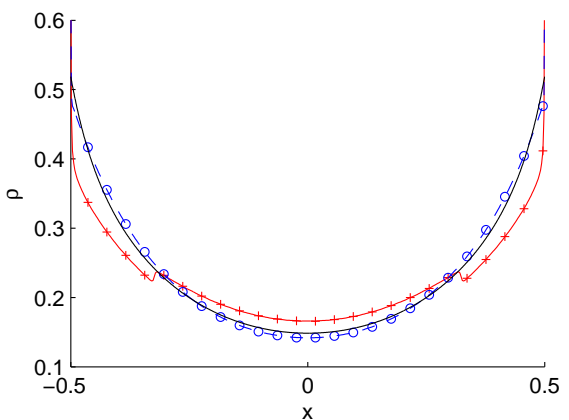

(c) $N=3$

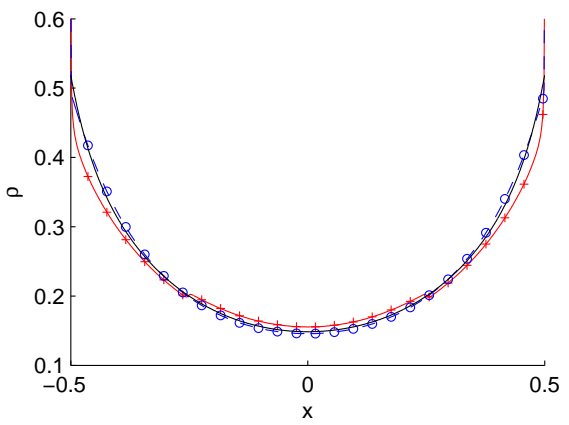

(e) $N=5$

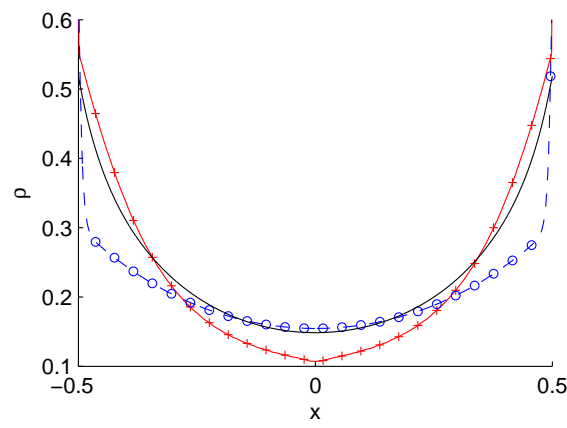

(b) $N=2$

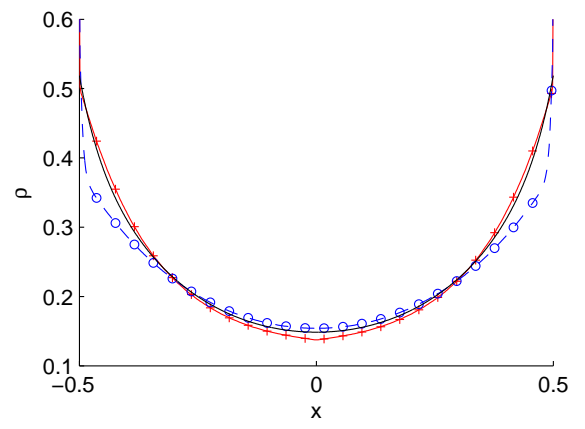

(d) $N=4$

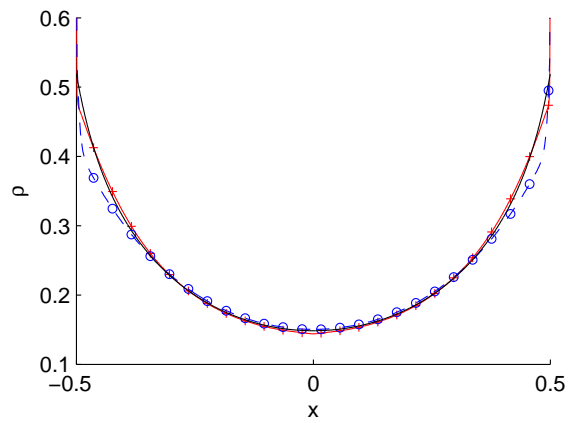

(f) $N=6$

Fig. 4.6. Red crosses are $M_{N}$. Blue circles are $P_{N}$. Solid, unadorned black line is the exact steady-state solution. The reader is referred to online version for color interpretation.

We note that the even- $N$ models also give rise to transient shocks. To show this, we display the $M_{2}$ and $M_{4}$ profiles at time $t=1.0$ in Figure 4.2. Like the odd cases, these two shocks appear after the beams reach the center of the domain. However, 


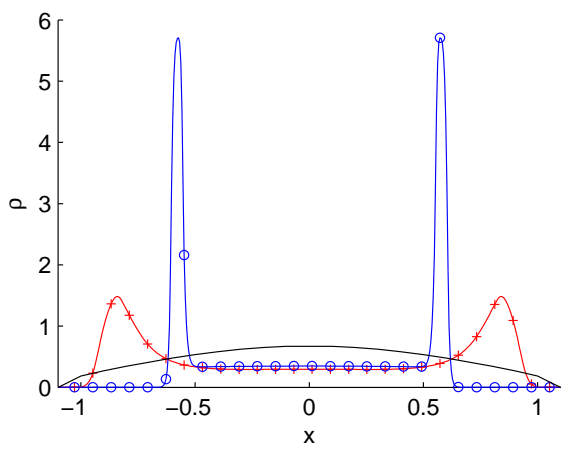

(a) $N=1$

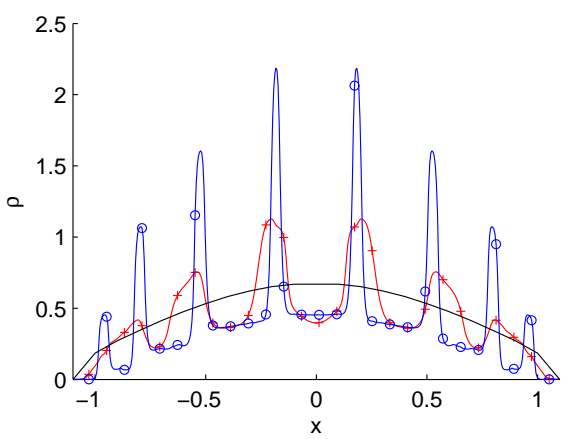

(c) $N=7$

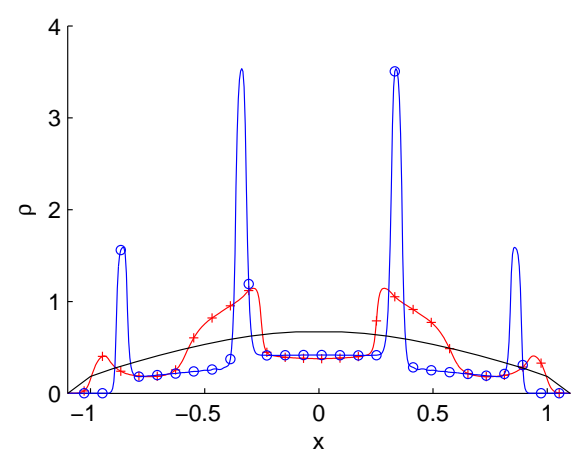

(b) $N=3$

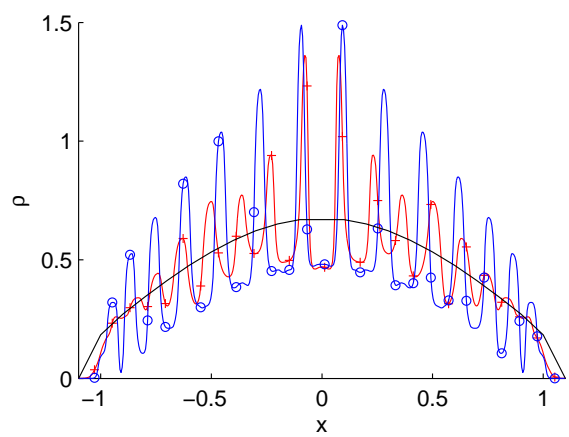

(d) $N=15$

Fig. 4.7. Planesource solution at $t=1.0$. Red crosses are $M_{N}$. Blue circles are $P_{N}$. Solid black is the semi-analytic solution. The reader is referred to online version for color interpretation.

they then disappear as the beams approach the boundary. The $M_{2}$ shock appears in the figure at $x= \pm 2.7$. The smaller $M_{4}$ shock appears at $x= \pm 3.0$. One can also see the formation of the center dip, particularly in the $M_{2}$ case. In addition both profiles include other transient oscillations which decay as the solution approaches steady state.

It is not entirely clear whether some of the structures observed in the even- $N$ solutions are physical or if they are numerical artifacts caused by small errors that arise when solving the dual optimization problem (2.11). In particular, we are concerned with oscillations in the transient profiles and the center dip in both the transient and steady-state solutions. As a first step to understanding these structures, we performed a high-resolution calculation for the case $N=2$, using a grid with 1600 spatial cells and 80 quadrature points. To better resolve local features, we apply a 10-point Gauss-Legendre quadrature on 8 congruent subintervals of $[-1,1]$, rather than an 80-point quadrature on the entire interval. ${ }^{4}$ Plots of the transient and steady-

${ }^{4}$ Such resolution is extremely expensive and adaptive quadrature will likely be required for any 


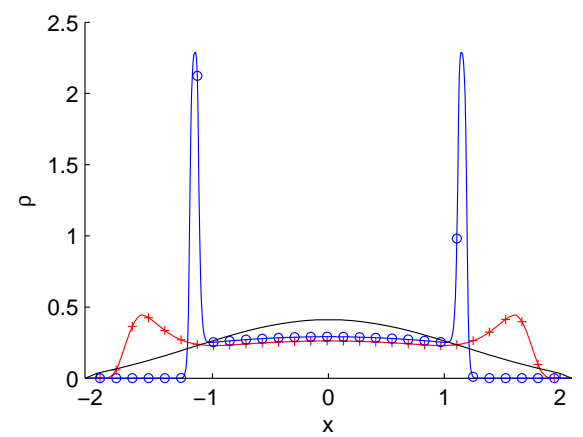

(a) $N=1$

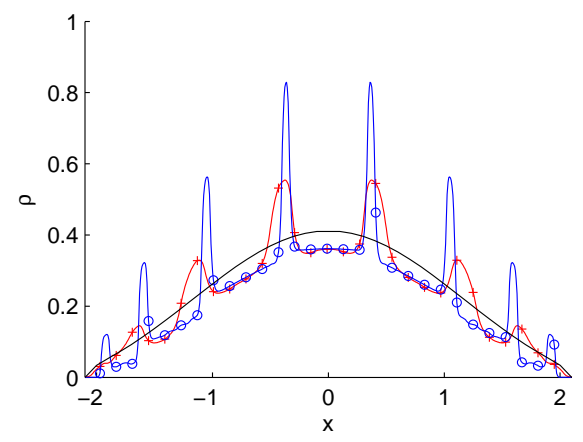

(c) $N=7$

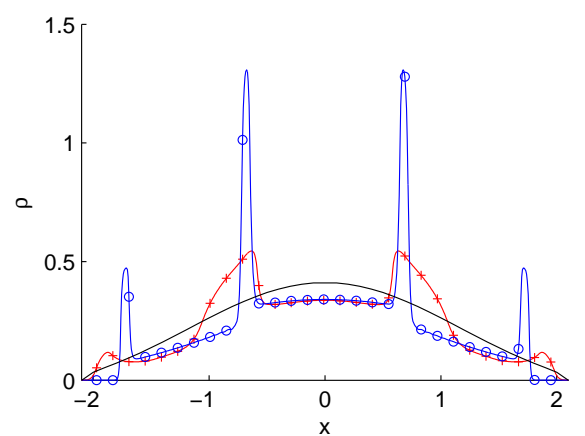

(b) $N=3$

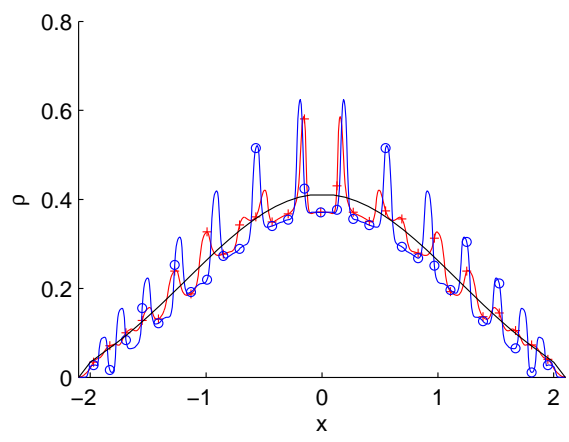

(d) $N=15$

FIG. 4.8. Planesource solution at $t=2.0$. Red crosses are $M_{N}$. Blue circles are $P_{N}$. Solid black is the semi-analytic solution. The reader is referred to online version for color interpretation.

state solutions are given in Figure 4.3 and 4.4, respectively. From these pictures, we conclude that the standard-resolution solutions (400 cells, 20 quadrature points) are nearly converged. The oscillations in the transient solution are less pronounced in the high-resolution case, but they still appear. Hence no definitive conclusions can be made about their origin. On the other hand, the center dip, although small in the steady-state case, persists and appears to be more than just an artifact of the numerics. As further evidence of this fact, we include the steady-state profile for the second moment $\Sigma:=\left\langle m_{2} \psi\right\rangle=\left\langle 0.5\left(3 \mu^{2}-1\right)\right\rangle$ in Figure 4.5. The center dip in $\rho$ corresponds to a bump in $\Sigma$ that is easier to identify.

In Figure 4.6 we compare the $M_{N}$ solutions with standard $P_{N}$ solutions, which are computed in exactly the same way except that we replace $G_{j}$ in (3.5) by $\mathbf{c}_{j}^{T} \mathbf{m}$, where

$$
\mathbf{c}_{j}=\left\langle\mathbf{m m}^{T}\right\rangle^{-1} \mathbf{u}_{j}
$$

practical implementation. 


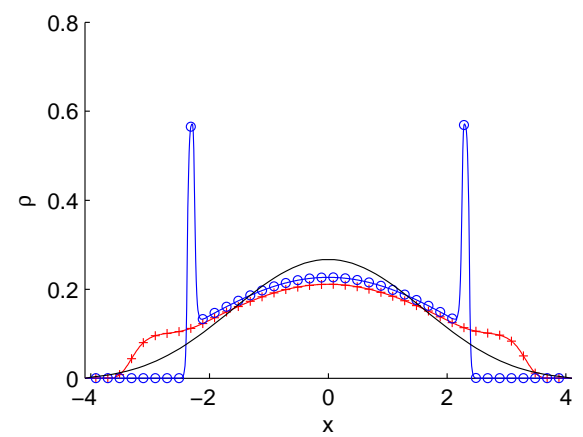

(a) $N=1$

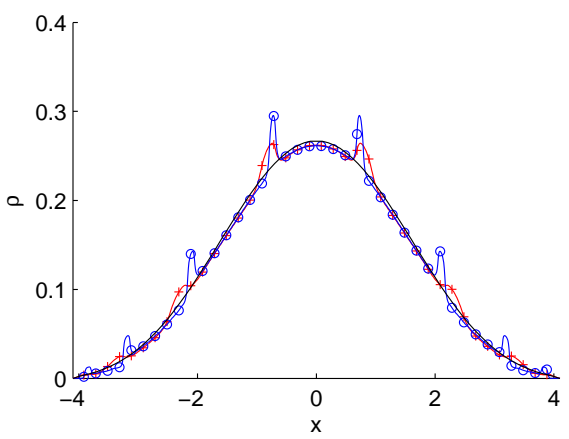

(c) $N=7$

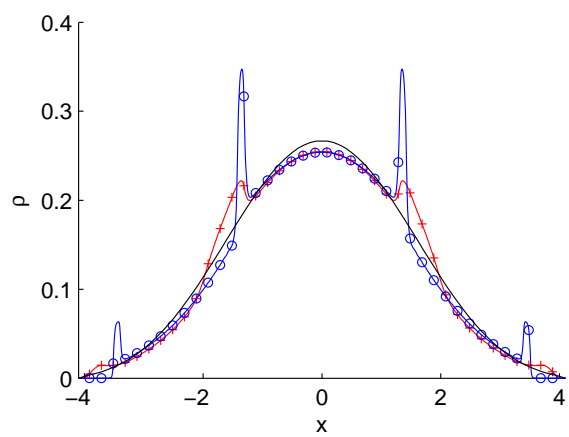

(b) $N=3$

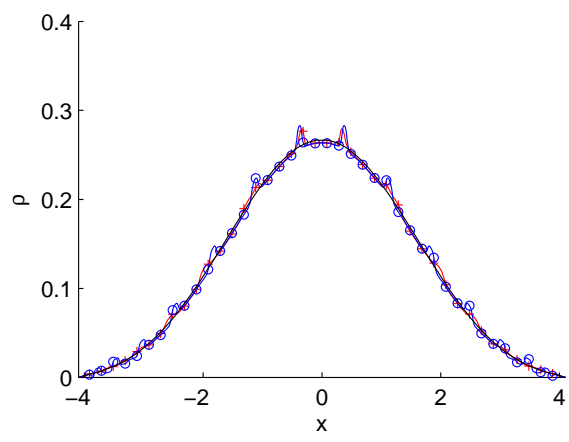

(d) $N=15$

FIG. 4.9. Planesource solution at $t=4$.0. Red crosses are $M_{N}$. Blue circles are $P_{N}$. Solid black is the semi-analytic solution. The reader is referred to online version for color interpretation.

The $P_{N}$ equations are linear and thus do not produce shocks. However, it is wellknown in the transport community (see, for example, [7]) that the $P_{N}$ equations for even $N$ are not accurate at boundaries. This fact is readily observed in Figure 4.6, where the even $M_{N}$ solutions are clearly better. For the odd case, the $P_{N}$ solutions are more accurate; however, this is not always the case. For the test case in [5], for example, it is noted that the $M_{1}$ solution is qualitatively wrong (because of the shock), but quantitatively better than the corresponding $P_{1}$ solution.

4.2. Plane source. In the plane-source problem, particles are emitted from an initial plane source into an infinitely long medium. The symmetry of this problem allows it to be represented in the one-dimensional setting, where $x$ measures the signed, normal distance to the plane. The plane-source problem is a torture test of a method's ability to handle very strong discontinuities. In the absence of any scattering, the $P_{N}$ method represents the transport solution as a series of delta functions emanating from the initial source. Any scattering in the problem serves to smooth out these delta functions [4].

The plane source has been simulated with the $M_{1}$ closure in [5]. There it was found 


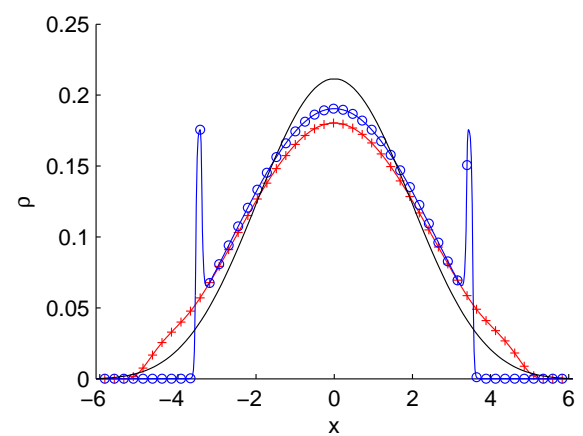

(a) $N=1$

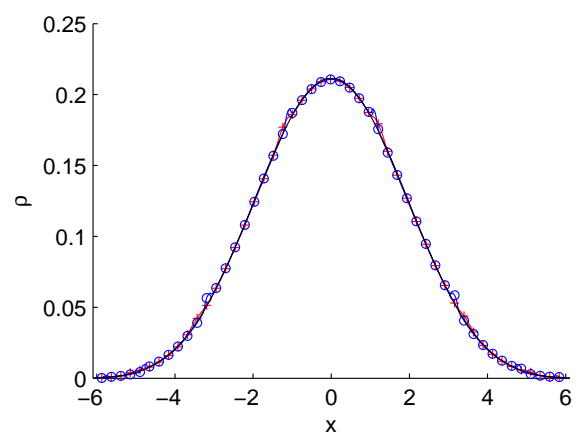

(c) $N=7$

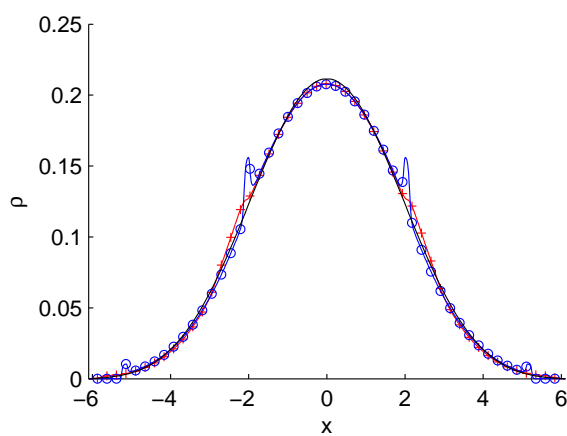

(b) $N=3$

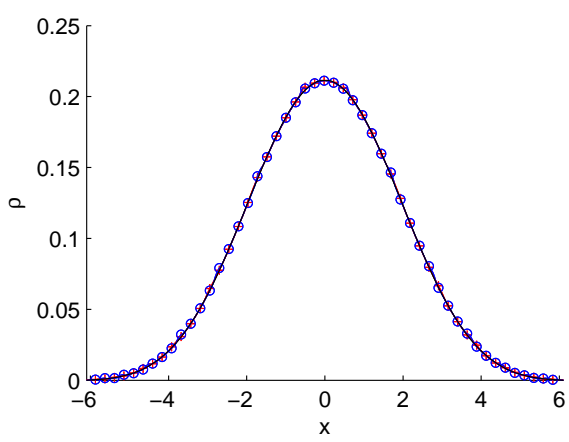

(d) $N=15$

FIG. 4.10. Planesource solution at $t=10.0$. Red crosses are $M_{N}$. Blue circles are $P_{N}$. Solid black is the semi-analytic solution. The reader is referred to online version for color interpretation.

that the non-physical wave effects (from the delta functions) in $P_{N}$ closures were less pronounced in the $M_{1}$ system. Moreover the $M_{1}$ system could move particles away from the source at peak speed (here equal to one), while particle speeds in linear $P_{N}$ systems are limited by the maximum eigenvalue of the flux matrix. For loworder closures, this value is significantly smaller than the maximum particle velocity: $1 / \sqrt{3} \simeq 0.577$ for $P_{1}$ and approximately 0.861 for $P_{3}$. As $N$ increases, the maximum eigenvalue eventually approaches one. Generally speaking, the $P_{N}$ approximation is better for (i) longer times, (ii) larger scattering rates, and (iii) larger values of $N$.

We implement the plane-source problem assuming a purely scattering material with $\sigma_{\mathrm{t}}=\sigma_{\mathrm{a}}=1.0$, with no source $(S=0)$ and initial condition

$$
F_{0}(x, \mu)=0.5 \delta(x)+F_{\text {floor }},
$$

where $F_{\text {floor }}=5.0 \times 10^{-5}$. Again a small, positive baseline value is used to maintain stability of the scheme, which is otherwise compromised by small errors in the solution of the optimization problem (2.11). Numerically, the delta function is represented with cell average values $0.25 / \Delta x$ in the two center cells with a common edge at $x=0$. 
Although the problem is defined on an infinite domain, boundary conditions must be implemented in practice. To do so, we define a computational domain $[-L / 2, L / 2]$, where $L=2 t_{\text {final }}+0.2$. This choice of $L$ is large enough to ensure that the domain contains non-negligible features of the solution. At the endpoints, we apply the boundary condition

$$
F_{1}(x, t)=F_{2}(x, t)=F_{\text {floor }}
$$

We choose the spatial mesh size $\Delta x$ based on the criteria that $\Delta x^{2} / L$ be roughly constant. For $t_{\text {final }}=1.0,2.0,4.0$, and 6.0 , this leads to $\Delta x=0.005,0.007,0.01$, and 0.0135 , respectively.

Results for the plane source are given in Figure 4.7-4.10 at several different times and for several choices of $N$. A semi-analytic solution [13] is also provided as a benchmark. We make a conclusion similar to the one in [5] - namely, that the wave effects in the $M_{N}$ profiles are less pronounced that in the $P_{N}$ profiles. For the most part, the $M_{N}$ profiles are qualitatively and quantitatively better than the $P_{N}$ profiles.

\section{Conclusions and discussion}

In this paper, we have presented computations of entropy-based $\left(M_{N}\right)$ models for a linear transport equation with slab geometry. These computations were performed using a kinetic scheme that is formally second-order in space and can be first or second order in time.

The key difficulty in our computations is in solving the dual optimization problem for the coefficients $\boldsymbol{\alpha}$. Our algorithm, which uses the built in MATLAB function fminunc, does fairly well in most cases, but has trouble in regimes where the underlying kinetic distribution is beam-like. Even so, we are able to generate interesting results for two important test problems.

We conclude from our results that $M_{N}$ models of all order may produce unphysical shocks, although the magnitude of such shocks becomes smaller as the size of the system increases. We observe also that, for even values of $N$, the $M_{N}$ models behave better than the $P_{N}$ models near the boundaries of the domain in the two-beam problem. However, it is still questionable in this case whether $M_{N}$ models produce better results than $P_{N}$ models, which are cheaper to simulate on serial or moderately parallel architectures. For the plane-source problem, it appears that the $M_{N}$ models yield better results than $P_{N}$.

Future work in this area is focused mainly on solving the dual problem. It should be noted that some numerical work already exists; see, for example, $[2,18]$ and references therein. While MATLAB provides an excellent interface for learning, the computational effort required for the dual problem limits experimentation. Thus it will be necessary in the future to implement the scheme with a compiled language. Future efforts also include parallel implementation, which, as discussed in the Introduction, is one of the motivating factors for studying entropy-based methods.

\section{REFERENCES}

[1] International Exascale Software Project Roadmap, 2009.

[2] R. Abramov, The multidimensional maximum entropy moment problem: A review on numerical methods, Commun. Math. Sci., 8, 377-392, 2009.

[3] A. Anile, W. Allegretto and C. Ringhofer, Mathematical Problems in Semiconductor Physics, Lecture Notes in Mathematics, Springer-Verlag, Berlin, 1823, 2003. Lectures given at the C.I.M.E. Summer School held in Cetraro, Italy on July 15-22, 1998. 
[4] T.A. Brunner, Riemann Solvers for Time-Dependent Transport Based on the Maximum Entropy and Spherical Harmonics Closures, PhD thesis, University of Michigan, 2000.

[5] T.A. Brunner and J.P. Holloway, One-dimensional Riemann solvers and the maximum entropy closure, J. Quant Spect. and Radiative Trans, 69, 543-566, 2001.

[6] R. Dautray and J.L. Lions, Mathematical Analysis And Numerical Methods For Science And Technology, Volume 6: Evolution Problems II, Spinger-Verlag, Berlin, 2000.

[7] B. Davison, Neutron Transport Theory, Oxford University Press, London, 1973.

[8] S.M. Deshpande, Kinetic theory based new upwind methods for inviscid compressible flows, American Institute of Aeronautics and Astronautics, New York, 86-0275, 1986.

[9] W. Dreyer, M. Herrmann and M. Kunik, Kinetic solutions of the Boltzmann-Peierls equation and its moment systems, Continuum Mechanics and Thermodynamics, 16, 453-469, 2004.

[10] B. Dubroca and J.L. Fuegas, Étude théorique et numérique d'une hiérarchie de modèles aus moments pour le transfert radiatif, C.R. Acad. Sci. Paris, I. 329, 915-920, 1999.

[11] B. Dubroca and A. Klar, Half-moment closure for radiative transfer equations, J. Comput. Phys., 180, 584-596, 2002.

[12] M. Frank, B. Dubroca and A. Klar, Partial moment entropy approximation to radiative heat transfer, J. Comput. Phys., 218, 1-18, 2006.

[13] B.D. Ganapol, Homogeneous infinite media time-dependent analytic benchmarks for X-TM transport methods development, tech. rep., Los Alamos National Laboratory, March, 1999.

[14] S. Gottlieb and C.W. Shu, Total variation diminishing Runge-Kutta schemes, Math. Comput., 67, 73-85, 1998.

[15] C. Groth and J. McDonald, Towards physically realizable and hyperbolic moment closures for kinetic theory, Continuum Mechanics and Thermodynamics, 2009.

[16] A. Harten, P.D. Lax and V. Leer, On upstream diferencing and Godunov-type schemes for hyperbolic conservation laws, SIAM Rev., 25, 35-61, 1983.

[17] C.D. Hauck, C.D. Levermore and A.L. Tits, Convex duality and entropy-based moment closures: Characterizing degenerate densities, SIAM J. Control Optim., 47, 1977-2015, 2008.

[18] W. Huang, Heuristic solutions to polynomial moment problems with some convex entropic objectives, Numerical Algorithms, 12, 297-308, 1996.

[19] S. Jin and C.D. Levermore, Numerical schemes for hyperbolic systems of conservation laws with stiff diffusive relaxation, J. Comput. Phys., 126, 449-467, 1996.

[20] M. Junk, Domain of definition of Levermore's five moment system, J. Stat. Phys., 93, 11431167, 1998.

[21] M. Junk, Maximum entropy for reduced moment problems, Math. Mod. Meth. Appl. S., 10, 1001-1025, 2000.

[22] M. Junk and V. Romano, Maximum entropy moment system of the semiconductor Boltzmann equation using Kane's dispersion relation, Continuum Mechanics and Thermodynamics, 17, 247-267, 2005.

[23] E.W. Larsen and C.G. Pomraning, The $P_{N}$ theory as an asymptotic limit of transport theory in planar geometry - I: Analysis, Nucl. Sci. Eng, 109, 49-75, 1991.

[24] E.W. Larsen and C.G. Pomraning, The $P_{N}$ theory as an asymptotic limit of transport theory in planar geometry - II: Numerical results, Nucl. Sci. Eng., 109, 76-85, 1991.

[25] C.D. Levermore, Boundary conditions for moment closures, Presented at Institute for Pure and Applied Mathematics University of California, Los Angeles, CA on May, 27, 2009.

[26] C.D. Levermore, Moment closure hierarchies for kinetic theory, J. Stat. Phys., 83, 1021-1065, 1996.

[27] E.E. Lewis and J.W.F. Miller, Computational Methods in Neutron Transport, John Wiley and Sons, New York, 1984.

[28] R.B. Lowrie and J.E. Morel, Methods for hyperbolic systems with stiff relaxation, Int. J. Numer. Meth. Fluids, 40, 413-423, 2002.

[29] P.A. Markowich, C.A. Ringhofer and C. Schmeiser, Semiconductor Equations, Springer-Verlag, New York, 1990.

[30] S.A. McKee, W.A. Wulf and T.C. Landon, Bounds on memory bandwidth in streamed computations, Euro-Par '95: Proceedings of the First International Euro-Par Conference on Parallel Processing, London, UK, Springer-Verlag, 83-99, 1995.

[31] G.N. Minerbo, Maximum entropy Eddington factors, J. Quant. Spectrosc. Radiat. Transfer, 20, 541-545, 1978.

[32] P. Monreal and M. Frank, Higher order minimum entropy approximations in radiative transfer, preprint.

[33] B. Perthame, Boltzmann type schemes for gas dynamics and the entropy property, SIAM J. on Numer. Anal., 27, 1405-1421, 1990.

[34] B. Perthame, Second-order Boltzmann schemes for compressible euler equations in one and 
two space dimensions, SIAM J. Numer. Anal., 29, 1-19, 992.

[35] G.C. Pomraning, Variational boundary conditions for the spherical harmonics approximation to the neutron transport equation, Ann. Phys., 27, 193-215, 1964.

[36] G.C. Pomraning, Radiation Hydrodynamics, Pergamon Press, New York, 1973.

[37] S.L. Rosa, G. Mascali and V. Romano, Exact maximum entropy closure of the hydrodynamical model for Si semiconductors: The 8-moment case, SIAM J. Appl. Math., 70, 710-734, 2009.

[38] J. Schneider, Entropic approximation in kinetic theory, Math. Model. Numer. Anal., 38, 541$561,2004$.

[39] C.W. Shu and S. Osher, Effcient implementation of essentially non-oscillatory shock capturing schemes, J. Comp. Phys., 77, 439-471, 1988.

[40] H. Struchtrup, Kinetic schemes and boundary conditions for moment equations, Z. Angew. Math. Phys., 51, 346-365, 2000.

[41] D. Wright, M. Frank and A. Klar, The minimum entropy approximation to the radiative transfer equation, preprint.

[42] W.A. Wulf and S.A. McKee, Hitting the memory wall: implications of the obvious, SIGARCH Comput. Archit. News, 23, 20-24, 1995. 